\title{
Fraturas Ósseas em uma Unidade de Cuidados Intensivos Neonatais
}

\author{
Bone Fractures in a Neonatal Intensive Care Unit
}

\author{
Ângela MACHADO ${ }^{1}$, Gustavo ROCHA ${ }^{1}$, Ana Isabel SILVA ${ }^{2}$, Nuno ALEGRETEE ${ }^{3,4}$, Hercília GUIMARÃES 2,5 \\ Acta Med Port 2015 Mar-Apr;28(2):204-208
}

RESUMO

Introdução: As fraturas ósseas são raras durante o período neonatal. Algumas das fraturas, sobretudo as de ossos longos, podem ocorrer durante o parto. Por outro lado, os recém-nascidos internados em Unidades de Cuidados Intensivos Neonatais têm um risco aumentado de fraturas ósseas por várias razões.

Objetivo: Avaliar a incidência e caracterizar as fraturas ósseas nos recém-nascidos internados numa Unidade de Cuidados Intensivos Neonatais nível III.

Material e Métodos: Análise retrospetiva dos recém-nascidos internados na Unidade de Cuidados Intensivos Neonatais com o diagnóstico na alta de uma ou mais fraturas ósseas, entre janeiro de 1996 a junho de 2013.

Resultados: Oitenta recém-nascidos apresentaram uma ou mais fraturas ósseas. Em 76 (95\%) recém-nascidos, as fraturas foram atribuídas a traumatismo do parto. A fratura mais comum foi da clavícula em 60 (79\%) recém-nascidos, seguida da fratura craniana em $6(8 \%)$ recém-nascidos. Em dois $(2,5 \%)$ recém-nascidos, prematuros de extremo baixo peso, as fraturas foram interpretadas como decorrentes de osteopenia da prematuridade. Ambos apresentaram fraturas múltiplas, um dos quais de várias costelas.

Conclusão: Uma mudança das práticas obstétricas, aliada à melhoria dos cuidados prestados ao recém-nascido prematuro contribuíram para a diminuição da incidência de fraturas ósseas no período neonatal. Contudo nos recém-nascidos prematuros o seu diagnóstico pode estar subestimado, dado o elevado risco de desenvolvimento de fratura que estes recém-nascidos apresentam.

Palavras-chave: Fracturas Ósseas/epidemiologia; Portugal; Recém-Nascido; Unidades de Cuidados Intensivos Neonatais.

\section{ABSTRACT}

Introduction: Fractures during the neonatal period are rare. Some fractures, especially long bones, may occur during birth. Moreover, neonates hospitalized in the Neonatal Intensive Care Unit have an increased risk of fractures for several reasons.

Objective: To evaluate the incidence and characterize fractures in newborns admitted in a tertiary Neonatal Intensive Care Unit.

Material and Methods: A retrospective analysis of the newborns admitted to the Neonatal Intensive Care Unit with a diagnosis at discharge of one or more bone fractures from January 1996 to June 2013.

Results: Eighty neonates had one or more fractures. In $76(95 \%)$ infants the fractures were attributed to birth injury. The most common fracture was the clavicle fracture in $60(79 \%)$ neonates, followed by skull fracture in $6(8 \%)$. In two $(2.5 \%)$ neonates, extremely low birth weight infants, fractures were interpreted as resulting from osteopenia of prematurity. Both had multiple fractures, and one of them with several ribs.

Conclusion: A change in obstetric practices allied to improvement premature neonate's care contributed to the decreased incidence of fractures in neonatal period. But in premature infants the diagnosis may be underestimated, given the high risk of fracture that these infants present.

Keywords: Fractures, Bone/epidemiology; Infant, Newborn; Intensive Care Units, Neonatal; Portugal.

\section{INTRODUÇÃo}

As fraturas ósseas são raras no período neonatal. ${ }^{1}$ No entanto, durante o parto podem ocorrer fraturas de ossos longos, nomeadamente da clavícula, associada a eventos como a distocia de ombros, bem como fraturas cranianas, na grande maioria dos casos decorrentes do uso de fórceps ou ventosa. ${ }^{2}$ Por outro lado, os recém-nascidos (RNs) internados em Unidades de Cuidados Intensivos Neonatais (UCIN) têm um risco aumentado de fraturas ósseas por várias razões incluindo prematuridade, baixo peso ao nascimento, malnutrição, traumatismo secundário a intervenções médicas bem como a efeitos colaterais de fárma$\cos ^{1-5}$ No RN pré-termo, a causa específica da fratura é geralmente difícil de estabelecer, sendo muitas vezes atribuída à osteopenia da prematuridade. ${ }^{1}$ A maioria dos casos são identificados acidentalmente em radiografias feitas por outras razões, pelo que a incidência exata das fracturas nos prematuros é desconhecida. ${ }^{2}$ De acordo com os poucos estudos publicados, a incidência das fraturas é muito variável, entre 1,2 a $10,5 \%$, sendo maior em RNs de muito baixo peso (MBP). ${ }^{1,6}$

O objetivo deste estudo foi avaliar a incidência e caracterizar as fraturas ósseas nos RNs internados numa UCIN nível III, com o propósito de otimizar as melhores práticas preventivas e terapêuticas.

\footnotetext{
1. Serviço de Neonatologia. Hospital Pediátrico Integrado. Centro Hospitalar de São João E.P.E. Porto. Portugal.

2. Serviço de Medicina Física e Reabilitação. Centro Hospitalar de São João E.P.E. Porto. Portugal.

3. Serviço de Ortopedia. Centro Hospitalar de São João E.P.E. Porto. Portugal.

4. Departamento de Ortopedia. Faculdade de Medicina. Universidade do Porto. Porto. Portugal.

5. Departamento de Pediatria. Faculdade de Medicina. Universidade do Porto. Porto. Portugal.

Recebido: 29 de Julho de 2014 - Aceite: 16 de Fevereiro de 2015 | Copyright @ Ordem dos Médicos 2015
} 


\section{MATERIAL E MÉTODOS}

Análise retrospetiva, através da consulta do processo clínico, de todos os RNs internados na nossa UCIN com o diagnóstico na alta de uma ou mais fraturas ósseas, entre janeiro de 1996 a junho de 2013. Foram analisadas as seguintes variáveis: idade gestacional; peso ao nascimento; sexo; tipo de parto; motivo de admissão; complicações neonatais, incluindo doença das membranas hialinas (DMH), displasia broncopulmonar (DBP), sépsis nosocomial, enterocolite necrosante (NEC), patência de canal arterial (PCA), hemorragia intraventricular (HIV), leucomalácia periventricular (LPV), e retinopatia da prematuridade (ROP); tratamento administrado como corticoides pós-natais, surfactante, diuréticos, ventilação mecânica invasiva, fototerapia e sedação.

\section{RESULTADOS}

No período de estudo, foram admitidos no serviço 7364 doentes. Destes, $80 \mathrm{RNs}$ apresentaram uma ou mais fraturas ósseas, correspondendo a uma prevalência de 1,1\%.

Em 76 (95\%) RNs as fraturas foram atribuídas a traumatismo do parto (Tabela 1). A fratura mais comum foi a da clavícula em $60(79 \%)$ RNs. Na maioria (97\%) o parto foi por via vaginal, tendo sido aplicada ventosa em $31 \%$. Verificou-se lesão do plexo braquial em $20 \%$ dos RNs, e todos evoluíram sem sequelas. Contudo, nenhum destes RNs foi admitido por apresentar fratura da clavícula. O motivo de admissão na UCIN foi variado: malformação congénita (incluindo cardiopatia) $(n=15)$, patologia infeciosa $(n=14)$, patologia respiratória $(n=8)$, patologia cirúrgica $(n=6)$, asfixia perinatal $(n=5)$, icterícia $(n=3)$, outros $(n=9)$.

A segunda fratura associada a traumatismo do parto mais observada foi a fratura craniana em $6(8 \%)$ RNs. A maioria $(n=5)$ nasceu por ventosa, um dos quais convertido em cesariana por tentativa frustada de extração fetal. Destes, todos apresentaram hemorragia subdural ou subaracnoideia, exceto o RN cujo parto foi convertido em cesariana. Um destes casos, admitido em coma por choque hipotensivo com acidose metabólica, evoluiu rapidamente para falência multiorgânica acabando por falecer. Outro $\mathrm{RN}$, que iniciou convulsões nas primeiras horas de vida, evoluiu com paralisia cerebral. Os restantes evoluíram favoravelmente, sem sequelas.

Relativamente aos RNs com outras fraturas associadas a traumatismo de parto, verificou-se a morte de um RN prematuro de 34 semanas com encefalopatia hipóxico-isquémica por falência multiorgânica no qual foi identificada fratura do úmero após o parto.

Foram admitidos dois $(2,5 \%)$ RNs com fratura craniana resultante de traumatismo cranioencefálico (TCE) decorrente de queda no domicílio (Tabela 2). Um destes RN (caso 1, Tabela 2) apresentava hemorragia subaracnoideia extensa com evolução rápida para choque hipovolémico e morte. O outro caso (caso 2, Tabela 2) apresentava hemorragia subdural, e iniciou convulsões no terceiro dia após o TCE. Este RN evoluiu com paralisia cerebral.

Nos restantes dois $(2,5 \%) \mathrm{RNs}$, prematuros de extremo baixo peso, as fraturas foram interpretadas como decorrentes de osteopenia da prematuridade (Tabela 3). Num des-

Tabela 1 - Fraturas por traumatismo de parto $(n=76)$

\begin{tabular}{|c|c|}
\hline Caraterística & Valor \\
\hline \multicolumn{2}{|l|}{ Sexo } \\
\hline Feminino & $34(45 \%)$ \\
\hline Masculino & $42(55 \%)$ \\
\hline Idade gestacional (mediana) & 39 semanas ( $23-42$ semanas) \\
\hline \multirow{2}{*}{$\begin{array}{l}\text { Peso ao nascimento (mediana) } \\
\text { Macrossomia }\end{array}$} & $3345 \mathrm{~g}(806-5250 \mathrm{~g})$ \\
\hline & $13 \%$ \\
\hline \multicolumn{2}{|l|}{ Tipo parto } \\
\hline Eutócico & $45(59 \%)$ \\
\hline Ventosa & $24(32 \%)$ \\
\hline Cesariana & $6(8 \%)$ \\
\hline Fórceps & $1(1 \%)$ \\
\hline \multicolumn{2}{|l|}{ Fratura } \\
\hline Clavícula & $60(79 \%)$ \\
\hline Parietal & $5(7 \%)$ \\
\hline Occipital & $1(1 \%)$ \\
\hline Úmero & $4(5 \%)$ \\
\hline Fémur & $3(4 \%)$ \\
\hline Costelas & $2(3 \%)$ \\
\hline Septo nasal & $1(1 \%)$ \\
\hline Duração internamento & 6 dias $(1-82$ dias $)$ \\
\hline Óbito & $2(3 \%)$ \\
\hline
\end{tabular}


Tabela 2 - Fraturas por TCE $(n=2)$

\begin{tabular}{lll}
\hline Caraterística & Valor & \\
& Caso 1 & Caso 2 \\
\hline Sexo & Feminino & Masculino \\
Idade gestacional ao nascimento & 39 semanas & 40 semanas \\
Peso ao nascimento & $2850 \mathrm{~g}$ & $3348 \mathrm{~g}$ \\
Dias de vida ao diagnóstico & 19 dias & 7 dias \\
Fratura & Parietal & Parietal \\
Duração internamento & 1 dia & 13 dias \\
Óbito & Sim & Não \\
\hline
\end{tabular}

tes casos (caso 1, Tabela 3) em D103 de vida, por presença de tumefação ao nível da coxa esquerda, foi diagnosticada uma fratura do fémur e, subsequentemente de várias costelas após realização de radiografia do esqueleto. No outro caso (caso 2, Tabela 3) em D57 de vida, foi constatada fratura do fémur bilateralmente, e úmero e rádio esquerdos, e em D144 de vida do úmero direito por radiografia realizada em contexto de intercorrência infeciosa. À data do diagnóstico das referidas fraturas, ambos os RNs apresentavam elevação da fosfatase alcalina (FA) (caso 1: $775 \mathrm{U} / \mathrm{L}$; caso 2: $456 \mathrm{U} / \mathrm{L}$ ) e níveis baixos de fósforo $(\mathrm{P})$ (caso 1: $3,4 \mathrm{mg} /$ $\mathrm{dL}$; caso 2: 2,5 mg/dL). Os níveis séricos de cálcio $(\mathrm{Ca})$ eram normais. No caso 2, foi também doseada a paratormona (PTH) que se encontrava elevada $(320 \mathrm{pg} / \mathrm{mL})$ e a vitamina $D$ estava normal $(21,6 \mathrm{ng} / \mathrm{mL})$.

\section{DISCUSSÃO}

A maioria das fraturas identificadas esteve relacionada o traumatismo do parto. A fratura da clavícula foi a mais comum, o que está de acordo com a literatura. Ocorre espontaneamente em aproximadamente $0,4-10 \%$ dos partos vaginais, e raramente em cesarianas. ${ }^{7,8} \mathrm{O}$ fator de risco mais consistente é o peso ao nascimento elevado, particularmente $>4000 \mathrm{~g}^{8,9}$ Outros fatores de risco associados são um comprimento superior a $52 \mathrm{~cm}$, um segundo estadio do trabalho de parto prolongado, ${ }^{7}$ partos instrumentalizados $^{7,8}$ e idade materna mais avançada. ${ }^{8}$ Este tipo de fratura está também associada à distocia de ombros, , 4,7-9 verificada no nosso estudo em apenas dois (3\%) RNs.

A incidência de fratura da clavícula foi muito elevada na nossa amostra, no entanto, estes RNs foram admitidos na unidade por outros motivos, não sendo esta fratura habitualmente observada em UCIN. Os RNs com este tipo de fratura raramente têm sintomas, e geralmente têm uma evolução sem sequelas a longo-prazo. Contudo, cerca de $9 \%$ apresentam concomitantemente lesão do plexo braquial. ${ }^{7} \mathrm{Na}$ nossa amostra, a percentagem de lesão do plexo foi superior $(20 \%)$.

As fraturas do úmero são as segundas fraturas de ossos longos mais comuns no RNs, ${ }^{10}$ tal como no nosso estudo. Este tipo de fraturas, bem como as do fémur estão muitas vezes associadas a cesarianas, durante a extração dos membros. ${ }^{10-13}$ Contudo, nós verificamos apenas um RN com fratura do úmero que nasceu de cesariana, sendo que nos três restantes, o parto foi eutócico mas com extrações referidas como difíceis. O mesmo se verificou nas fraturas de fémur, em que apenas um dos três casos foi parto por cesariana.

Relativamente às fraturas cranianas, a incidência na nossa amostra foi de $7,5 \%(n=6)$, sendo a fratura óssea mais comum atribuída ao parto a seguir à fratura da clavícula. A instrumentalização do parto é um fator de risco importante para este tipo de lesão, bem como, um aumento da incidência de hemorragia intracraneana, $4,14,15$ como se verificou nos nossos resultados. Em cinco (83\%) dos RNs com fratura craniana foi aplicada ventosa, e quatro $(67 \%)$ apresentaram hemorragia subdural ou subaracnoideia. Segundo a literatura, a incidência deste tipo de traumatismo tem vindo a diminuir devido a mudanças nas práticas obstétricas. ${ }^{16} \mathrm{~A}$ prevalência de fraturas cranianas numa coorte retrospetiva em que foi efetuada radiografia do crânio em todos os casos de aplicação de ventosa, foi de $5 \% .{ }^{17}$ No entanto, todas foram fraturas lineares em RNs assintomáticos. Curiosamente, quatro dos nossos casos ocorreram durante o último semestre do estudo.

As fraturas cranianas no período neonatal podem resultar de traumatismo de outra etiologia, como nos dois $(2,5 \%)$ RNs admitidos na nossa UCIN após queda no domicílio. Cerca de 1 a $3 \%$ das pequenas quedas podem provocar fratura craniana, e muito raramente, a fratura pode estar associada a uma hemorragia subdural de contato. ${ }^{18}$ Neste tipo de fratura, independentemente da etiologia, a evolução e sequelas a longo-prazo dependem principalmente dos danos cerebrais diretos do traumatismo. ${ }^{14}$

Nos RNs, fraturas das costelas, especialmente das costelas posteriores, são raras e altamente suspeitas de resultarem de lesões não acidentais. ${ }^{1,2}$ Este tipo de fratura associada ao traumatismo de parto é ainda mais raro, e verifica-se em RNs grandes com extrações difíceis. ${ }^{1,19} \mathrm{Na}$ nossa amostra obtivemos, surpreendentemente, dois (2,5\%) RNs com fraturas de costelas associadas a traumatismo de parto. Um caso por ventosa e no outro caso um parto por fórceps. Nenhum destes RNs foi sujeito a manobras de reanimação cardiorrespiratória, outra causa extremamente rara de fraturas de costelas nesta idade, embora comum nos adultos. ${ }^{1,19}$ 
Tabela 3 - Fraturas por osteopenia da prematuridade $(n=2)$

\begin{tabular}{|c|c|c|}
\hline Caraterística & $\begin{array}{l}\text { Valor } \\
\text { Caso } 1\end{array}$ & Caso 2 \\
\hline Sexo & Masculino & Masculino \\
\hline Idade gestacional & 28 semanas & 31 semanas \\
\hline Peso ao nascimento & $880 \mathrm{~g}$ & $506 \mathrm{~g}$ \\
\hline SGA & Não & Sim \\
\hline DMH & Severa & Ligeira \\
\hline Surfactante & 3 doses & 1 dose \\
\hline DBP & Sim & Sim \\
\hline Corticóides pós-natal (duração) & 12 dias & 13 dias \\
\hline Diuréticos (duração) & 69 dias & 7 dias \\
\hline VM invasiva (duração) & 185 dias & 147 dias \\
\hline Sépsis nosocomial (hemocultura positiva) & $\operatorname{Sim}$ & Sim \\
\hline $\mathrm{NEC} \geq 2 \mathrm{~A}$ & Não & Não \\
\hline PCA sintomático & Sim & Não \\
\hline HIV $\geq$ III \pm enfarte & Não & $\operatorname{Sim}$ \\
\hline LPV & Não & Não \\
\hline ROP grau $\geq 2$ & Não & Não \\
\hline Fototerapia (duração) & 6 dias & 7 dias \\
\hline Sedação (duração) & 38 dias & 32 dias \\
\hline Duração internamento & 184 dias & 147 dias \\
\hline Óbito & Sim & Sim \\
\hline
\end{tabular}

SGA: pequeno para a idade gestacional; DMH: doença de membranas hialinas; DBP: displasia broncopulmonar; VM: ventilação mecânica, NEC: enterocolite necrosante; PCA: patência de canal arterial; HIV: hemorragia intraventricular; LPV: leucomalácia periventricular; ROP: retinopatia da prematuridade.

Contudo as fraturas no período neonatal podem estar associadas as outras etiologias, sobretudo em recém-nascidos prematuros internados em UCIN. Aproximadamente $80 \%$ da mineralização óssea ocorre durante o terceiro trimestre da gravidez, pelo que os RNs prematuros têm um risco aumentado de osteopenia. ${ }^{3}$ Particularmente aqueles que nascem < 28 semanas de gestação, com baixo peso ao nascimento e/ou cujo período neonatal imediato é complicado com necessidade de nutrição parentérica prolongada, enterocolite necrosante, hiperbilirrubenemia conjugada, displasia broncopulmonar e uso de furosemida. ${ }^{2,3}$

A doença óssea metabólica da prematuridade (DOMP), nova designação da osteopatia da prematuridade, ocorre em cerca de $23 \%$ dos RNs com MBP e em $55-60 \%$ nos RNs de extremo baixo peso (EBP).,6 Não existe nenhum metabolito que isoladamente possa ser considerado marcador de DOMP. ${ }^{4}$ Os níveis de Ca e $\mathrm{P}$ podem ser normais apesar dos baixos níveis de reserva, devido ao efeito da PTH. ${ }^{4,6} \mathrm{O}$ marcador mais amplamente aceite da DOMP é o aumento da FA, no entanto os valores de cut-off variam muito entre os estudos, desde $300 \mathrm{IU} / \mathrm{L}$ (nível máximo considerado normal no RN) a $900 \mathrm{IU} / \mathrm{L} .^{1,5} \mathrm{~A}$ incidência de fraturas em RNs de EBP reportada é de $10 \%$, mas atualmente será menor. ${ }^{6} \mathrm{Na}$ nossa série, apenas foram identificados dois casos, RNs de EBP, cujas fraturas foram atribuídas à DOMP. Neste grupo é mais comum encontrar fraturas múltiplas, sobretudo das costelas, ${ }^{1}$ tal como na nossa amostra, em que os dois casos apresentavam fraturas múltiplas, um dos quais de várias costelas.

A diminuição da incidência de fraturas neste grupo deve-se principalmente à melhoria dos cuidados prestados aos RNs prematuros. ${ }^{2} \mathrm{O}$ aporte adequado de $\mathrm{Ca}$ e $\mathrm{P}$ na alimentação parentérica, bem como a minimização da sua duração; a fortificação do leite materno; o uso de fórmulas específicas para prematuros; a redução do uso de corticóides e diuréticos; a suplementação precoce com vitamina $D$; e a mobilização dos membros; são atitudes cada vez mais implementadas nas UCIN, por forma a promover o aumento da massa óssea.

\section{CONCLUSÃO}

Apesar do declínio, segundo a literatura, da incidência dos traumatismos associados ao parto, resultado da melhoria das práticas obstétricas, as fraturas ósseas, sobretudo da clavícula, ainda permanecem uma causa frequente de morbilidade neonatal. Contudo o prognóstico das fraturas de ossos longos por traumatismo de parto é habitualmente favorável.

Os RNs prematuros, sobretudo os EBP, têm risco mais elevado de desenvolver fraturas múltiplas, de etiologia habitualmente multifatorial. Contudo a incidência de fraturas neste grupo tem vindo a diminuir, principalmente devido à 
melhoria dos cuidados prestados a estes RNs. No entanto, a sua incidência pode estar subestimada, uma vez que a maioria das fraturas nos prematuros é assintomática e diagnosticada acidentalmente. Na nossa série, a baixa incidência de fraturas neste grupo de RNs também pode ser justificada por se tratar de um estudo retrospetivo. Assim é fundamental prevenir a DOMP, mas também a detetar precocemente, através da doseamento periódico, principalmente, dos níveis séricos de FA e $\mathrm{P}$, por forma a instituir o seu tratamento, e desta forma prevenir as fraturas.

\section{REFERÊNCIAS}

1. Wei C, Stevens J, Harrison S, Mott A, Warner J. Fractures in a tertiary neonatal intensive care unit in Wales. Acta Paediatr. 2012;101:587-90.

2. Bishop N, Sprigg A, Dalton A. Unexplained fractures in infancy: looking for fragile bones. Arch Dis Child. 2007;92:251-6.

3. Herald AL, Butler S, Mactier H, McDevitt H, Young D, Ahmed SF. Prevalence and characteristics of rib fractures in ex-preterm infants. Pediatrics. 2012;130:1116-9.

4. Aloy JF, Álvarez-Domínguez E, Pérez-Fernández JM, Moretones-Suñol G, Vidal-Sicart S, Botet-Mussons F. Metabolic bone disease and bone mineral density in very preterm infants. J Pediatr. 2014;164:499-504.

5. Vachharajani AJ, Mathur AM, Rao R. Metabolic bone disease of prematurity. NeoReviews. 2009;10:402-11.

6. Gomella TL, Cunningham MD, Eyal FG. Neonatology: management, procedures, on-call problems, diseases, and drugs. $6^{\text {th }}$ ed. Philadelphia: McGraw-Hill Companies; 2009.

7. Mavrogenis A, Mitsiokapa EA, Kanellopoulos AD, Ruggieri P, Papagelopoulos PJ. Birth fracture of the clavicle. Adv Neonatal Care. 2011;11:328-31.

8. Zdener TO, Engin-ustun Y, Aktulay A, Turkcapar F, Oguz S, Yapareyi EG, et al. Clavicular fracture: its prevalence and predisposing factors in term uncomplicated pregnancy. Eur Rev Med Pharmacol Sci. 2013;17:126972.

9. Hsu TY, Hung FC, Lu YJ, Ou CY, Roan CJ, Kung FT, et al. Neonatal clavicular fractures: clinical analysis on incidence, predisposing factors,

\section{CONFLITOS DE INTERESSE}

Os autores declaram a inexistência de quaisquer conflitos de interesse.

\section{FONTES DE FINANCIAMENTO}

Os autores declaram a inexistência de quaisquer fontes de financiamento externo.

diagnosis, and outcome. Am J Perinatol. 2002;19:17-21.

10. Sherr-Lurie N, Bialik GM, Ganel AG, Schindler A, Givon U. Fractures of the humerus in the neonatal period. Isr Med Assoc J. 2011;13:363-6.

11. Sabat D, Maini L, Gautam VK. Neonatal separation of distal humeral epiphysis during caesarean section: a case report. J Orthop Surg. 2011;19:376-8.

12. Basha A, Amarin Z, Abu-Hassan F. Birth-associated long-bone fractures. Int J Gynaecol Obstet. 2013;123:127-30.

13. Givon U, Sherr-Lurie N, Schindler A, Blankstein A, Ganel A. Treatment of femoral fractures in neonates. Isr Med Assoc J. 2007;9:28-9.

14. Doumouchtsis SK, Arulkumaran S. Head injuries after instrumental vaginal deliveries. Curr Opin Obstet Gynecol. 2006;18:129-34.

15. Doumouchtsis SK, Arulkumaran S. Head trauma after instrumental births. Clin Perinatol. 2008;35:69-83.

16. Wegner E, Bernstein IM. Operative vaginal delivery. UptoDate 2014; [consultado 2014 Jun 2]. Disponível em: www.uptodate.com.

17. Simonson C, Barlow P, Dehennin N, Sphel M, Toppet V, Murillo D, et al. Neonatal complications of vacuum-assisted delivery. Obstet Gynecol. 2007;109:626-33.

18. Case ME. Accidental traumatic head injury in infants and young children. Brain Pathol. 2008;18:583-9.

19. Rijn RR, Bilo RA, Roben SG. Birth-related mid-posterior rib fractures in neonates: a report of three cases (and a possible fourth case) and a review of the literature. Pediatr Radiol. 2009; 39:30-4. 
Ângela MACHADO, Gustavo ROCHA, Ana Isabel SILVA, Nuno ALEGRETE, Hercília GUIMARÃES

\section{Fraturas Ósseas em uma Unidade de Cuidados Intensivos Neonatais}

Acta Med Port 2015:28:204-208

Publicado pela Acta Médica Portuguesa, a Revista Científica da Ordem dos Médicos

Av. Almirante Gago Coutinho, 151

1749-084 Lisboa, Portugal.

Tel: +351218428 215

E-mail: submissao@actamedicaportuguesa.com

www.actamedicaportuguesa.com

ISSN:0870-399X | e-ISSN: 1646-0758

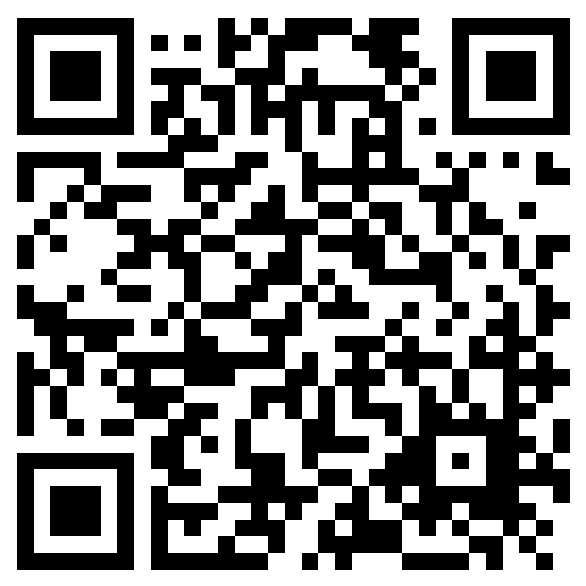

ACTA MÉDICA

PORTUGUESA 